\title{
Design and stress analysis of in-vessel saddle coils for MHD control in ASDEX Upgrade
}

\author{
T. Vierle ${ }^{\text {a }}$, B. Streibl ${ }^{\text {a }}$, M. Rott ${ }^{\text {a }}$, U. Seidel ${ }^{\text {a }}$, A. Herrmann ${ }^{\text {a }}$, \\ O. Neubauer ${ }^{\mathrm{b}}$, W. Suttrop ${ }^{\mathrm{a}}$, and the ASDEX Upgrade Team \\ a Max-Planck-Institut für Plasmaphysik, EURATOM Association, D-85740 Garching, \\ Germany \\ ${ }^{\mathrm{b}}$ Institut für Energieforschung - Plasmaphysik, Forschungszentrum Jülich GmbH, \\ Association EURATOM/FZJ, Trilateral Euregio Cluster, D-52425 Jülich, Germany
}

\begin{abstract}
A set of in-vessel saddle coils for MHD control in ASDEX Upgrade is described. A conventional coil design is chosen which employs a five turn square cross section copper conductor with central cooling bore embedded in an epoxy resin. The winding is enclosed in a solid metal casing, made of a thin deep-drawn inconel sheet in order to maximise the electrical resistance and minimise $L / R$ and shielding of the magnetic field. The bending stress in the coil casing due to the mount is reduced by reinforcing ribs perpendicular to the current direction. Fatigue stresses are avoided by application of a pre-stress on the casing exerted by the mounting bolts. Finite element stress calculations are performed to verify the design. Secondary stresses induced by thermal expansion in the casing and the conductor are well tolerable. The structural stiffness of the coil design provides a high resonance frequency, above the operation frequency range $f=\mathrm{DC} \ldots 1.2 \mathrm{kHz}$.
\end{abstract}

Key words: Tokamaks, ASDEX Upgrade, MHD control, in-vessel coils design PACS: 52.55 Fa, 07.05.Fb, 07.55.Db

\section{Introduction}

Magnetohydrodynamic instabilities in tokamaks can be controlled by external nonaxisymmetric magnetic field perturbations. To this end, a set of 24 in-vessel saddle coils is planned to be installed in ASDEX Upgrade. The coils are arranged in three toroidal rings of eight coils each in three different poloidal positions on the low field side. The upgrade will start with eight upper and eight lower coils ("B" coils)

Email address: Thomas.Vierle@ipp.mpg.de (T. Vierle). 


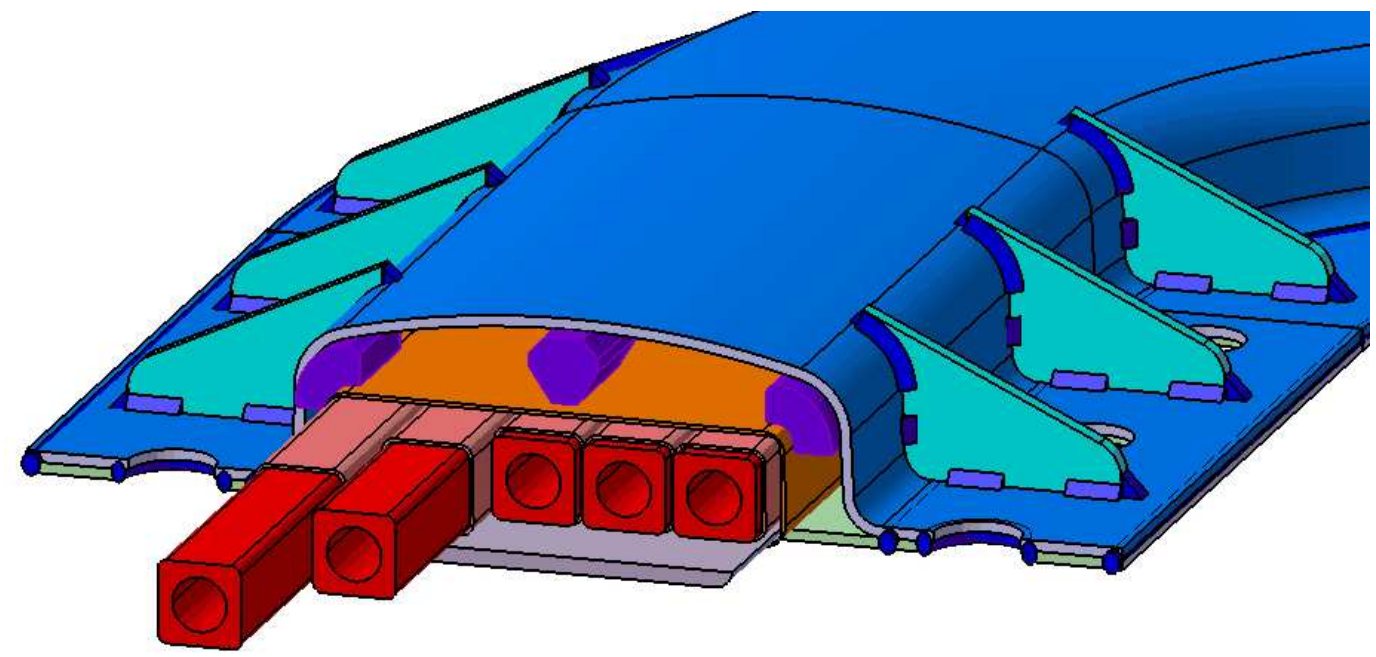

Fig. 1. 3D-view of the B-coil vertical (poloidal) branch, showing the arrangement of reinforcing ribs around densely spaced mounting holes.

and will later be complemented with eight middle coils ("A" coils), mounted around existing large vessel access ports and a planned additional conducting wall structure. This paper describes the mechanical design and design verification of the 16 B-coils. The physics objectives and overall project are introduced in [1]. Electromagnetic modeling of the coils' performance is described in [2].

The design principles are influenced by in-vessel coil designs on other fusion experiments, most notably the TEXTOR Dynamic Ergodic Divertor [3,4], DIII-D I-coils [5], and the W7-X control coils [6]. Like the latter, the B-coils employ water-cooled copper windings (multiple turns), embedded into a dielectric and are enclosed in a vacuum-proof metal casing. However the B-coils are designed to operate at frequencies up to $f=1.2 \mathrm{kHz}$ and therefore require care to minimise magnetic field shielding and inductive heating by eddy currents.

\section{Design description}

\subsection{In-vessel saddle coils}

Fig. 1 shows a 3-D view of a cutaway section of the B-coils. The winding (five turns of oxygen-free copper with central cooling water bore) is insulated with glass fabric tape around each individual turn and around the entire block. The winding block is fixed with spacers in a casing that consists of $1.2 \mathrm{~mm}$ thick inconel sheets. The casing top (facing towards the plasma) is deep-drawn, while the casing baseplate is cut from a flat sheet and only slightly bent to follow the contour of the mounting surface. 


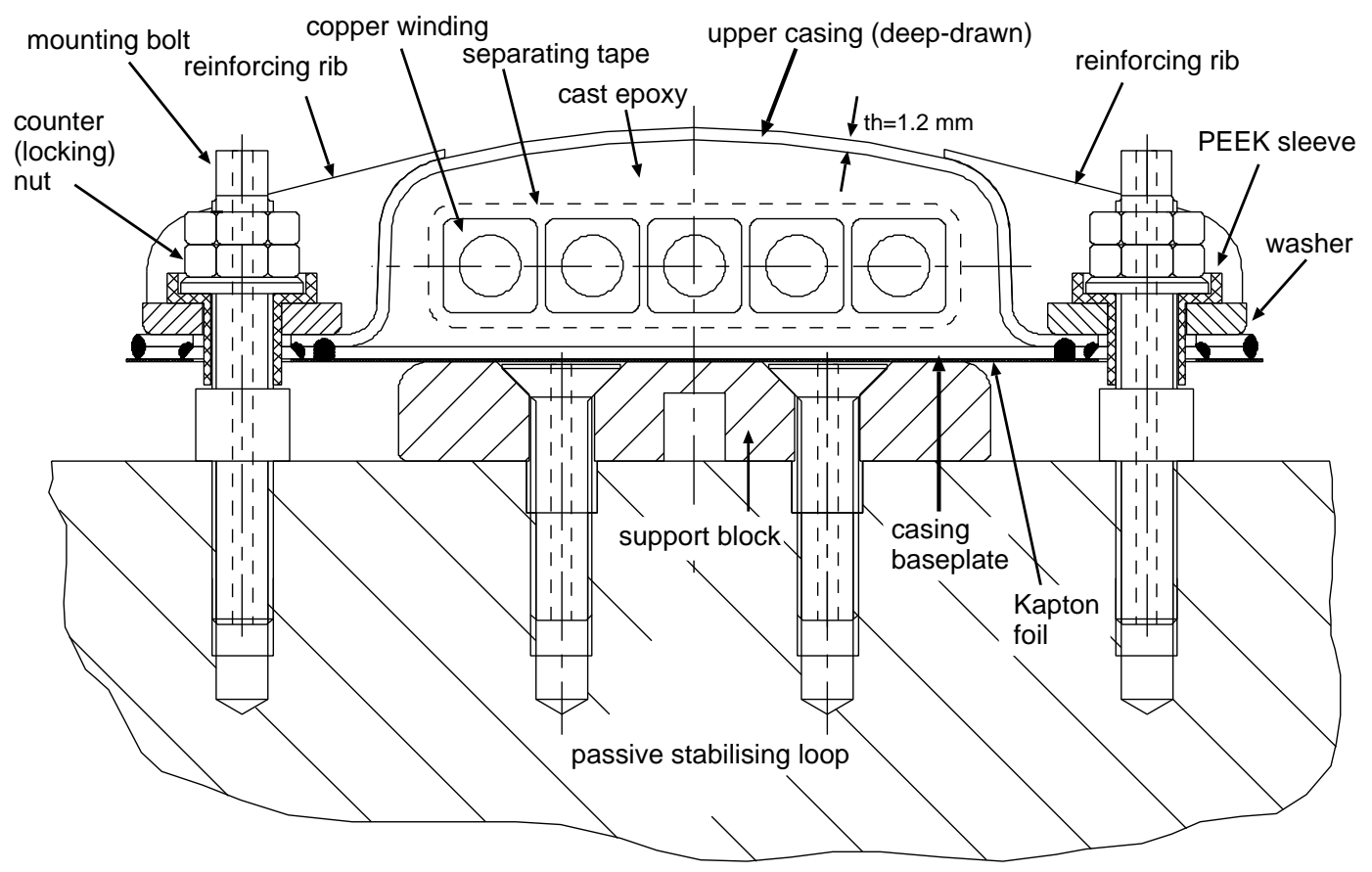

Fig. 2. Cross section of B-coil, mounted on the passive stabilising loop

Fig. 2 shows a cross section of the coil. Both casing sheets are welded with two line-welds at either side of the winding. The welds are applied to a separated region (welding tongue) in order to minimise heating of the coil interior during the welding process. Mounting holes are cut through the connected metal sheets. The casing top is reinforced with ribs against bending forces arising from the mount and electromagnetic forces on the conductors. The winding is immersed in an epoxy resin that withstands the baking temperature of ASDEX Upgrade, $150^{\circ} \mathrm{C}$. The epoxy also defines the maximum operating temperature of the coil, $90^{\circ} \mathrm{C}$. The coil insulation is wrapped outside with a thin separating tape to avoid bonding with the surrounding epoxy embedding.

The complete coils are mounted on the existing top and bottom passive stabilising loops (PSL), two massive toroidal copper conductors used to slow down the vertical plasma displacement for plasma position control. The distance to the PSL is defined by intermediate castellated support blocks (length: $26 \mathrm{~mm}$, thickness for upper Bcoils: $10 \mathrm{~mm}$, lower B-coils: $30 \mathrm{~mm}$ ). A large distance is preferred for low PSL image current and small magnetic field shielding [2]. However, the upper coils are mounted close to the PSL in order to minimise the restrictions on plasma shaping.

The coils are electrically connected with the PSL at one point and insulated otherwise to avoid bypass currents in the coil casing. The coils are mounted with prestress to avoid fatigue stresses in the steel casing due to coil currents with alternating polarity. Towards the plasma side, the coils are protected with graphite tiles. As part of the tungsten plasma facing components experiment in ASDEX Upgrade, the protecting tiles are coated with a thin $(3 \mu \mathrm{m})$ tungsten layer made by physical 


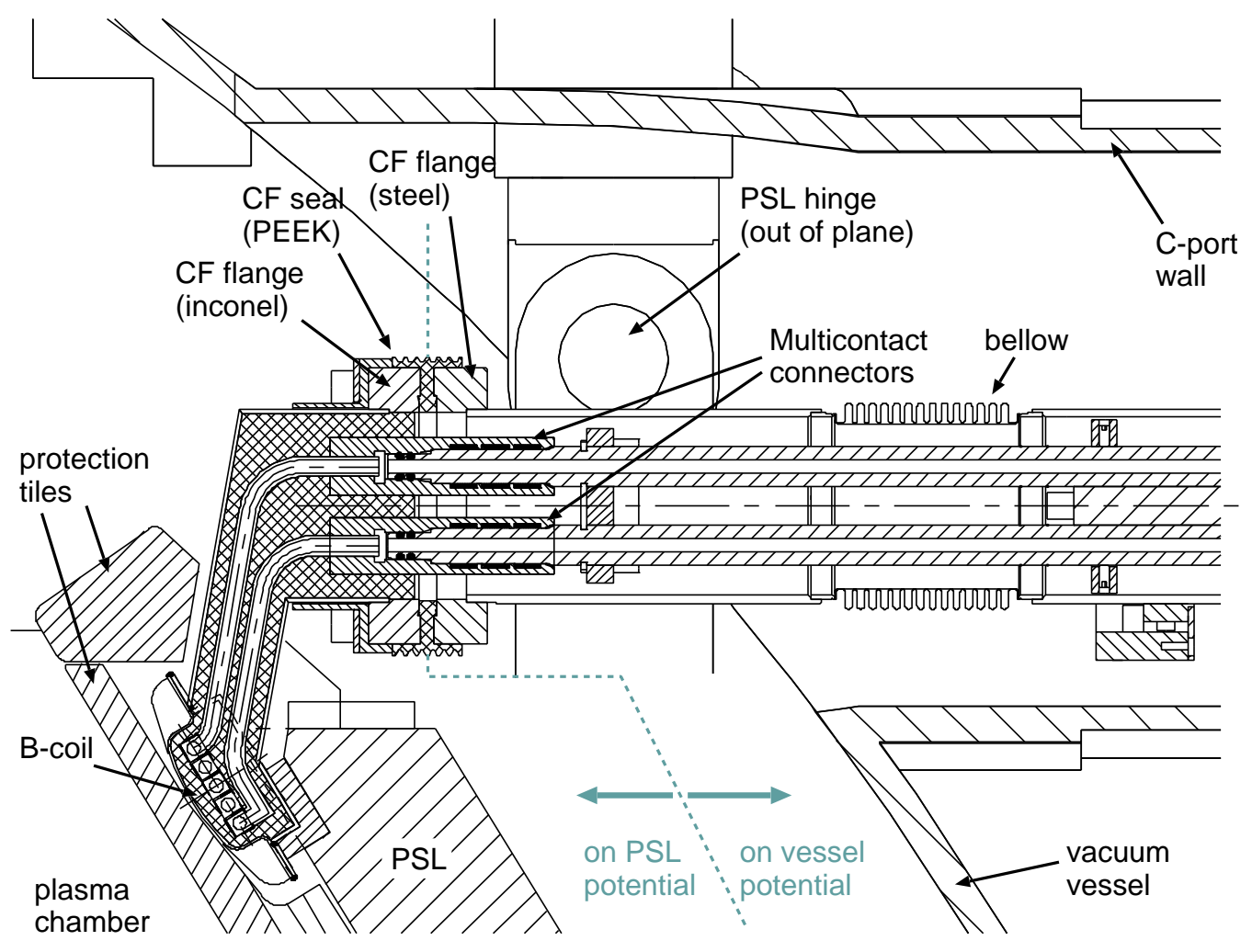

Fig. 3. Cross section of the coil-feedthrough connection region.

vapour deposition (PVD).

The stiffening rib of the coil casing reduces the bending stress at the transition from the lower to the upper casing shell. The bolt force presses the upper casing via the corner of the stiffener against the lower flat baseplate. The stiffener minimises the lateral movement of the vertical casing wall and thus introduces a larger pre-stress into the curved upper part of the casing, thereby increasing the compression of all insulation components. The combination of bolt pre-stress, stiffener and PSL support achieves a low stress level that stays constant within narrow limits as long as the magnetic force of a bolt period stays below the bolt pre-stress force. In this way, the structure is no longer subject to dynamic stress and can thus be rated for static conditions.

\subsection{Electrical and cooling water feedthrough}

The connection region of the upper coil and feedthrough of electrical and cooling water connections is shown in Fig. 3. The connection region is enclosed in a box of $2 \mathrm{~mm}$ thick steel sheets which is welded to the casing. The inner conductor end crosses the winding on the PSL side. In order to maintain the flat coil design, a machined PSL cut-out provides the space for the wire crossing and the surrounding enclosure. The electrical connection to the power feed and the cooling water 
connection are made by a plug/jack system, i.e. can be disconnected. This is necessary because the feedthrough unit is installed in the ASDEX Upgrade vacuum ports from the outside, while the coils are mounted from inside the vessel. Thus, no welding or soldering is necessary during the installation procedure. The high-voltage, high-current connectors use custom-made "multicontact" strips for low contact resistance. The coil-side connection unit (plug) has guide pins to facilitate plugging and is secured by lock bolts.

The conductor in the connection region is immersed in epoxy resin up to the base plate for the Multicontact connector. The plug itself is insulated by sleeves around the multicontacts. The flange gasket is a PEEK disk that also separates the electrical potential of PSL and vacuum vessel (potential difference $\leq 100 \mathrm{~V}$ ). The supply connections (electrical and cooling water) are made through ASDEX Upgrade upper and lower radial C-ports. Each coil spans two sectors, so that eight upper C-ports and eight lower C-ports will be used. The electrical and cooling water connection is made through a separate feeder tube. On the vessel side, this tube is attached to the outer flange of the C-port and mechanically supported inside the C-port. During normal operation, the feeder tube will be evacuated. The feeder tube is mechanically decoupled from the coil by a bellow, which takes up lateral and axial relative displacements of the connector and the tube, caused by tilting and vibrations of the PSL/coil assembly. The inner conductors allow the same lateral and axial displacement. The axial displacement is taken up at the outer C-port vacuum flange in a sliding seal of the feeder tube vacuum, made with two O-rings with grooves in the conductors, sliding on the insulating bushing. This is sufficient to accommodate the relative movement between PSL and vessel during baking ( $4 \mathrm{~mm}$ max.) and disruptions. The inner conductors are axially supported on the coil side at a fixed point in the tube between CF-flange and bellow. In lateral direction the inner conductor allows for a displacement of $2 \mathrm{~mm}$ in vertical ( $z$-) direction (bending over the full port length). The support disks in the feeder tube allow for lateral and axial play but take up the electromagnetic torque on the conductor pair.

\section{Design verification}

The coil design presented above has been validated in various ways. Performance parameters (magnitude and phase of the magnetic field produced), electrical load parameters, electromagnetic forces on active conductors, disruption-induced voltages and short-circuit currents, inductive heating of the passive structure and inbetween pulse re-cooling are discussed in Ref. [2]. Several straight mock-up coil and casing sections with reduced length but otherwise identical dimensions have been built to test the welding procedure (from different suppliers) and the fatigue life of the coil assembly. Fatigue tests with alternating loads have been performed for the poloidal coil leg $\left(l_{B s}=35 \mathrm{~mm}\right.$ bolt distance) for $10^{7}$ load cycles at the design load. This number of cycles is sufficient to validate the long-time fatigue strength 


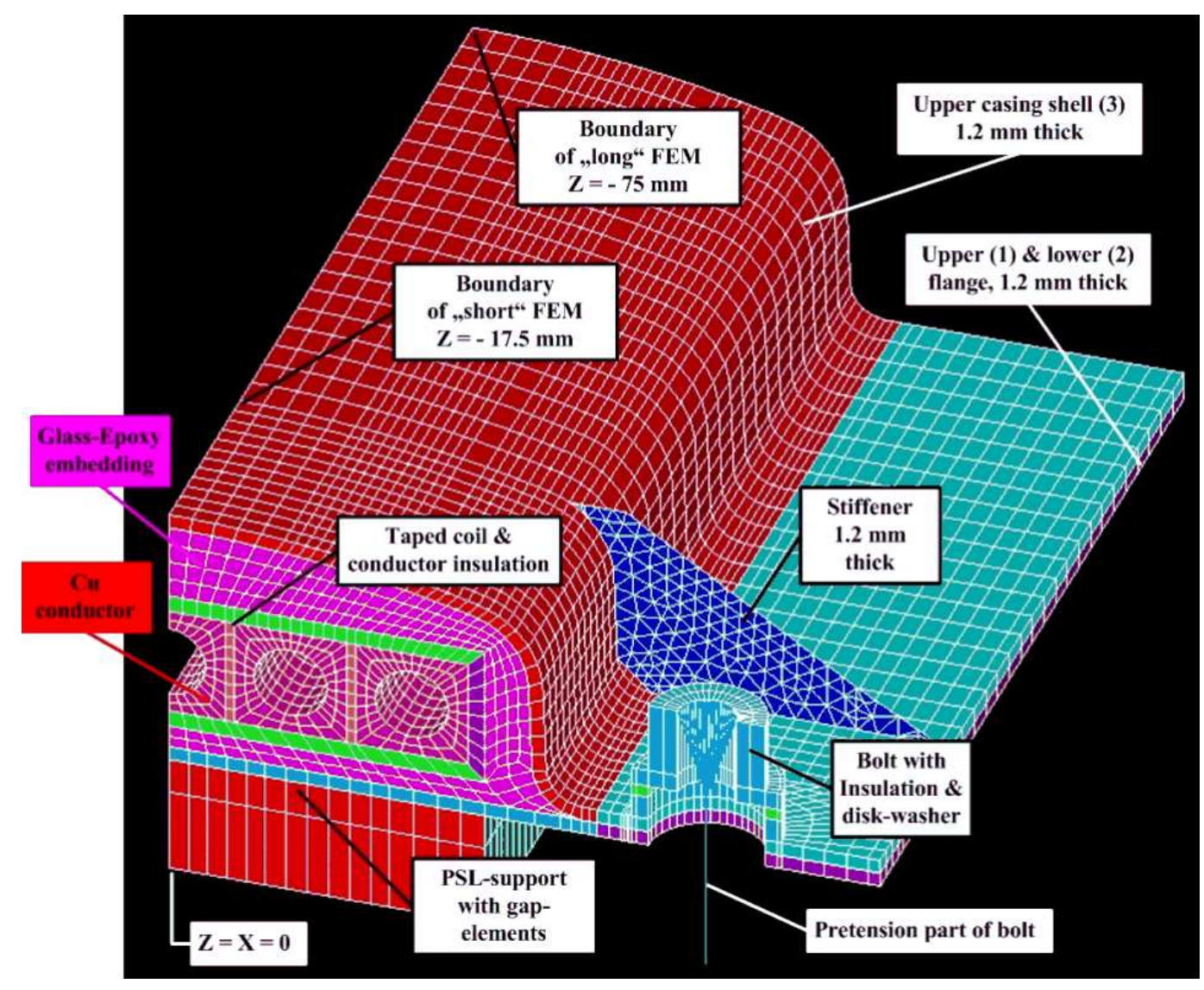

Fig. 4. Basic elements of the finite element model for the B-coil design as shown in Fig.1.

of the casing. Below we consider in more detail the finite element calculations performed to verify mechanical strength of the coil casing and the coil mount.

\subsection{Mechanical strength of coil casing}

The mechanical design is verified by $2 \mathrm{D}$ and $3 \mathrm{D}$ finite element calculations using the Ansys code. Two finite element models (FEMs) are used allowing for the different spacing of the bolt fixations for the poloidal $\left(l_{\mathrm{B}}=l_{\mathrm{Bs}}\right)$ and toroidal $\left(l_{\mathrm{B}}=l_{\mathrm{Bl}}\right)$ coil branches. The models, termed "short" and "long", respectively, approximate the coil by a straight section between bolt center with length equal to half the distance between bolts. Basic properties of the models are illustrated in Fig. 4 ("long" model). The long model has two stiffeners and the short model one stiffener per bolt period. Each conductor is meshed together with the insulation system and the coil embedding. The taped parts of the insulation for conductor and coil are merged, since their mechanical properties are identical. The different stiffness properties in the plane of the fabric layers and across them are taken into account.

The separation between epoxy and coil embedding in the casing is represented with Ansys "cement" elements, which transfer only compression forces and indicate if 
certain tension stress limits are exceeded, leading to cracks. This feature requires non-linear solution algorithms. Nonlinearities are also introduced by contact elements (called "gap" elements in Fig. 4), which transfer pressure loads and frictional shear only when the double nodes are in contact. Gap elements are applied between the disk washer of the bolt and the upper casing flange, also adjacent to the vacuum seal weld on the transition of upper and lower casing shells and on the support of the lower casing shell on the PSL. The bolt pre-stress is introduced by means of hinged bars (link-elements or trusses).

\subsection{Stress analysis results}

The pre-stress force per bolt is $F_{B}=600 \mathrm{~N}$. It provides a safety margin of a factor of two against the design load $\left(F_{R}\right)$, including a vertical plasma displacement with major disruption, and ensures favourable conditions for the assembly. Short and long FEM are evaluated separately; the largest stress value is quoted unless stated otherwise.

Because of the dominating toroidal field, the design line loads differ considerably for the poloidal leg $\left(f_{M R s}=17 \mathrm{kN} / \mathrm{m}\right)$ and toroidal coil leg $\left(f_{M R l}=3.4 \mathrm{kN} / \mathrm{m}\right)$. The axial bolt distances are adjusted to yield similar design forces, $F_{R s}=595 \mathrm{~N}$ and $F_{R l}=510 \mathrm{~N}$, respectively, for short and long bolt spacing.

The pre-stress force $F_{B}$ is simultaneously limited by bolt bending and case stressing. Thermal expansion of the toroidal housing leg introduces, via friction, lateral forces mainly into the bolts of the poloidal leg. In order to limit the resulting bending stress, the bolts are clamped at the PSL by a sleeve of increased diameter and with the largest possible height (see Fig. 2). In this way, the friction coefficient can become as large as about 0.3 without over-stressing the bolts.

The inconel material of the casing structure has a yield limit of $R_{p 02} \geq 200 \mathrm{MPa}$ and thus a stress limit of $S_{A}=2 / 3 R_{p 02} \geq 133 \mathrm{MPa}$. For the small FEM, Fig. 5 shows a typical fringe pattern of the equivalent stress $\left(S_{\mathrm{Eqv}}\right)$. The maximum $\left(M_{X}\right)$ occurs on the tip of the stiffener and amounts to $S_{\mathrm{Eqv}}=120 \mathrm{MPa}$. Over the whole operational regime this value stays constant within $3 \mathrm{MPa}$. Under the same conditions, the maximum $S_{\mathrm{Eqv}}=132 \mathrm{MPa}$ (pure bending stress) of the long FEM occurs on the flange hole. However, in this case the allowable stress would be the yield limit of $200 \mathrm{MPa}$.

The PSL support has a length of $26 \mathrm{~mm}$ for the toroidal legs. This length provides on the one hand sufficient space for its fixation and on the other hand does not require taking into account the toroidal curvature of the PSL. The short poloidal legs with their densely spaced mounting bolts use a continuous support. The compressive support stresses remain sub-critical $(\leq 25 \mathrm{MPa})$ over the complete operational range. Even a fourfold design load would not raise the pressure beyond $38 \mathrm{MPa}$ 


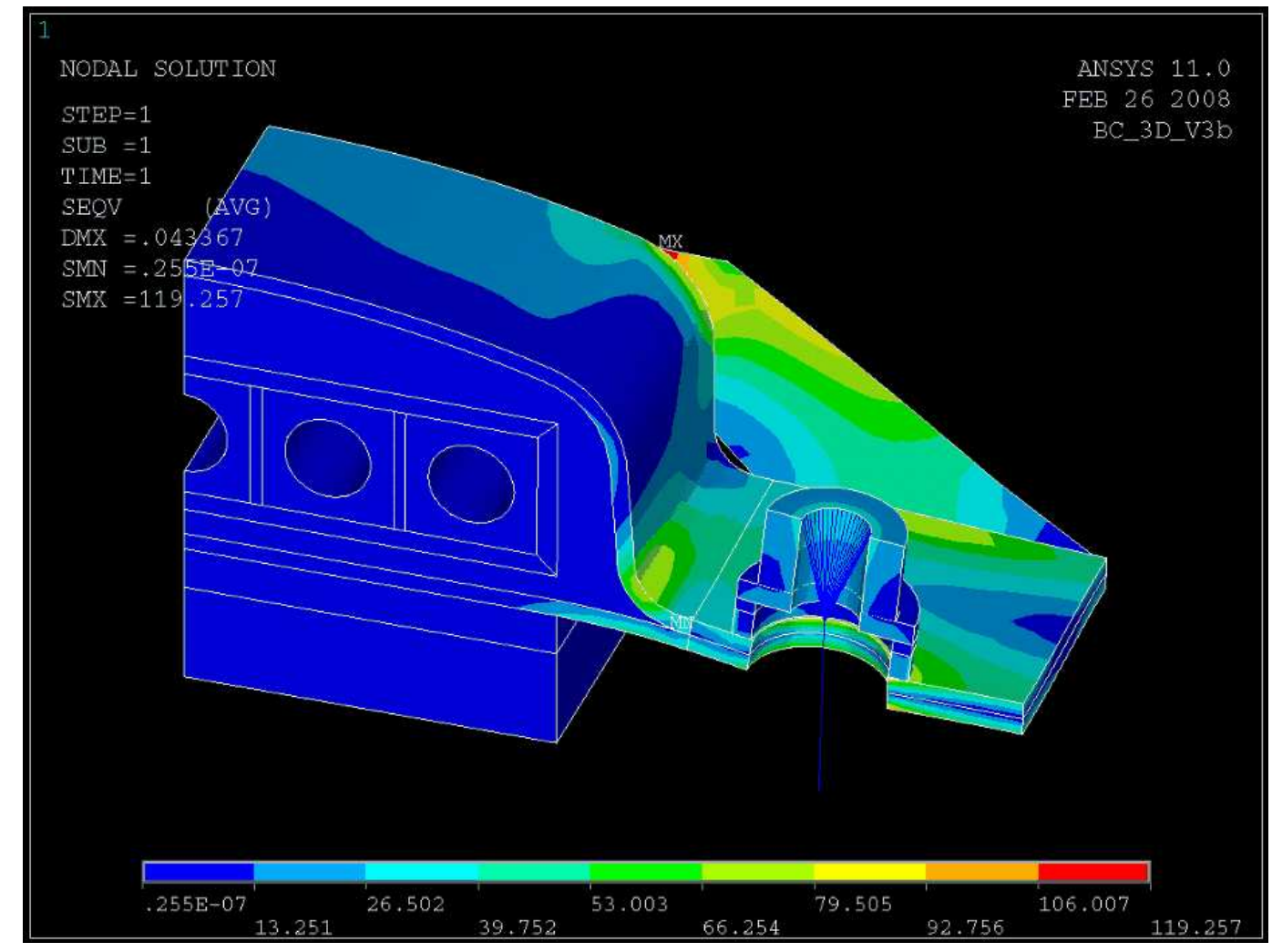

Fig. 5. Typical fringe pattern of the short FEM. The equivalent stress $S_{\text {Eqv }}$ is shown in false colours.

since the support area increases for larger downward forces. Underneath the discwasher of the bolt, the maximum pressure is $85 \mathrm{MPa}$ at the outer rim next to the stiffener. For a fourfold upward force, this increases to $145 \mathrm{MPa}$.

The coil insulation is strain limited by the steel casing. Its stresses are therefore of secondary nature. Within the operational range, the insulation strain stays a factor of 70 below the rupture strain $(2 \%)$. Under these conditions, cracks or delamination of the insulation can be excluded. In addition, thermal movements will not cause cracks since the embedding of the coil by glass granulate impregnated with epoxy is detached from the winding by a separating foil.

\subsection{Resonance frequency}

For the intended broad frequency range of the coils, the lowest major resonance frequency should be placed above the predicted corner frequency, $f_{C}=1.2 \mathrm{kHz}$ [2]. This leads to stiffness requirements, in particular for the toroidal leg, which has a significant unsupported length between bolts. Since the force gradient lengths are much longer than the bolt period, the longitudinal lowest frequency resonant mode can be regarded as that of a clamped beam with a homogenous line load. The equivalent beam stiffness is taken from the FE model. Assuming the mass to be 
concentrated in the symmetry plane, this provides a lower limit of the resonance frequency $\left(f_{\mathrm{R}, \min } \approx 2.2 \mathrm{kHz}\right)$. With the more realistic assumption of homogeneously distributed mass the upper limit is estimated as $\left(f_{\mathrm{R}, \max } \approx 3.6 \mathrm{kHz}\right)$. The vibration test of the simplified, straight mock-up with a bolt distance of $105 \mathrm{~mm}(140 \mathrm{~mm})$ has shown a resonance around $1.7 \mathrm{kHz}(1.2 \mathrm{kHz})$. These results indicate that resonance phenomena are unlikely to become important for the B-coil frequency range. When operated with switched power supplies, the switching frequency might be chosen to avoid hitting a resonance.

\section{Summary and Conclusions}

The ASDEX Upgrade in-vessel saddle coils (B-coils), as presented above, combine a robust design based on the proven example of the W7-X control coils with an extended frequency range from DC (static fields for ELM suppression, error field compensation) to about $f_{C}=1.2 \mathrm{kHz}$ (locked mode avoidance, resistive wall mode control). An hermetically sealed metal casing provides complete isolation of the winding from the torus vacuum. The thin casing sheet thickness mandatory for the high operation frequency results in high requirements for the structural design of the coil and its support on the passive stabilising loop. These needs are met by mounting the coils with significant pre-stress to avoid alternating stresses as well as narrow clamping distances and stiffeners to reduce bending stresses on the casing. As a result, a safety factor of two above the design forces is achieved which includes sufficient margin to accommodate additional forces from worst case disruptions. As such, the design is applicable for the middle coil set (A-coils) as well. However, a higher bandwidth of $3 \mathrm{kHz}$ is desired for the A-coils which is not achieved with the B-coil design. Therefore, two alternative concepts for the A-coils are currently investigated, a fully non-conducting, thermoplastic coil housing and a housing with sections of thin bellows separated by insulators.

\section{References}

[1] W. Suttrop, A. Herrmann, M. Rott, T. Vierle, U. Seidel, et al., In-vessel saddle coils for MHD control in ASDEX Upgrade, this conference.

[2] M. Rott, U. Seidel, B. Streibl, W. Suttrop, T. Vierle, et al., Electro-magnetic modeling of the planned active in-vessel coils for ASDEX Upgrade, this conference.

[3] B. Giesen, H. Bohn, W. Huettemann, O. Neubauer, M. Poier, et al., Fusion Eng. Design 37 (1997) 341.

[4] K. H. Finken, A. Abdullaev, W. Biel, M. F. M. de Bock, S. Brezinsek, et al., Contrib. Plasma Phys. 46 (2006) 515. 
[5] P. M. Anderson, C. B. Baxi, A. G. Kellman, E. E. Reis, and J. I. Robinson, Fusion Eng. Design 66-68 (2003) 791.

[6] T. Rummel and I. Schönewolf, Fusion Eng. Design 58-59 (2001) 47. 